\title{
Synergism between muramyl dipeptide and lipopolysaccharide in the inhibition of glycosaminoglycan synthesis in cultured rat costal chondrocytes
}

\author{
T Ikebe, M Hirata, F Yanaga, T Koga
}

\begin{abstract}
The effect of synthetic muramyl dipeptide on glycosaminoglycan synthesis in cultured rat costal chondrocytes was examined. Muramyl dipeptide alone had no effect on the glycosaminoglycan synthesis of rat chondrocytes, whereas Escherichia coli lipopolysaccharide and interleukin $1 \alpha$ inhibited glycosaminoglycan synthesis in a dose dependent manner. Muramyl dipeptide, when added to chondrocyte cultures in the presence of lipopolysaccharide, enhanced the lipopolysaccharide induced inhibition of glycosaminoglycan synthesis in a dose dependent manner. Adjuvant active analogues of muramyl dipeptide, but not adjuvant inactive analogues, also enhanced the lipopolysaccharide induced inhibition of glycosaminoglycan synthesis. In combination with muramyl dipeptide, to inhibit glycosaminoglycan synthesis, lipopolysaccharide could be replaced with the synthetic lipid $A$, an active principle of lipopolysaccharide. These results show that the muramyl dipeptide portion of bacterial peptidoglycan enhances the susceptibility of rat chondrocytes to the lipid A portion of bacterial lipopolysaccharide, and therefore the interaction between chondrocytes and bacterial cell wall components might be involved in damaging the cartilage in inflammatory joint diseases.
\end{abstract}

(Ann Rheum Dis 1993; 52: 32-36)

Destruction of the articular cartilage is one feature of inflammatory joint diseases such as rheumatoid arthritis. Bacterial cell wall components linked to the infection may trigger a breakdown in cartilage as lipopolysaccharide, a major component of the cell wall of Gram negative bacteria, stimulates macrophages to release interleukin 1 (IL-1) ${ }^{1}$ and tumour necrosis factor $\alpha{ }^{2}$ cytokines which promote cartilage breakdown and inhibit the synthesis of cartilage matrix. ${ }^{34}$ In addition, it seems likely that lipopolysaccharide itself enhances the cartilage breakdown and reduces re-synthesis through a direct mechanism. ${ }^{5}$

Peptidoglycan is a major cell wall component of bacteria. N-Acetylmuramyl-L-alanyl-D-isoglutamine or muramyl dipeptide is an essential component for a number of biological activities of bacterial cell wall peptidoglycan, including immunoadjuvant activity, ${ }^{6-10}$ induction of adjuvant arthritis, ${ }^{11}$ enhancement of collagen induced arthritis, ${ }^{12}$ bone resorption, ${ }^{13}$ stimula- tion of IL-1 production, ${ }^{14}$ and macrophage colony stimulating factor production. ${ }^{15} 16 \mathrm{We}$ reported that muramyl dipeptide inhibits glycosaminoglycan synthesis and promotes the release of glycosaminoglycan from the cell layer into the medium in cultures of rabbit costal chondrocytes, ${ }^{17}$ findings which suggested that muramyl dipeptide and lipopolysaccharide are important factors related to the destruction of cartilage.

We now report evidence that in rat chondrocytes muramyl dipeptide alone has no effect on glycosaminoglycan synthesis, yet it synergises with lipopolysaccharide in decreasing glycosaminoglycan synthesis when added to chondrocyte cultures simultaneously with or before lipopolysaccharide.

\section{Materials and methods}

MATERIALS

Muramyl dipeptide (Daiichi Pharmaceutical Co, Tokyo, Japan) and five synthetic muramyl dipeptide analogues were used; these analogues were synthesised as described elsewhere. ${ }^{18} 19$ Human recombinant IL- $1 \alpha\left(2.5 \times 10^{7} \mathrm{U} / \mathrm{mg}\right)$ was from Dainippon Pharmaceuticals (Osaka, Japan). Lipopolysaccharide derived from Escherichia coli $0 \cdot 55: \mathrm{B} 5$ and synthetic $E$ coli lipid A (506) were purchased from Difco Laboratories (Detroit, MI, USA) and Daiichi Pure Chemicals (Tokyo, Japan) respectively. Sulphur-35 labelled sulphate (carrier free) was from the Japan Atomic Energy Research Institute (Tokyo, Japan). Other materials used were commercial products of the highest grade available.

\section{CULTURE PROCEDURE}

Chondrocytes were obtained from the growth cartilage of ribs of four week old Wistar rats, as described by Shimomura et al. ${ }^{20}$ The isolated cells were seeded at a density of $1 \times 10^{5}$ cells/ microwell (16 mm diameter multiwell plate; Falcon, Oxnard, CA, USA) and maintained in Ham's F-12 medium (Nissui Pharmaceutical, Tokyo, Japan) supplemented with $10 \%$ fetal calf serum (heat inactivated at $56^{\circ} \mathrm{C}$ for 30 minutes) at $37^{\circ} \mathrm{C}$ in $95 \%$ air $/ 5 \%$ carbon dioxide.

ASSAY FOR GLYCOSAMINOGLYCAN SYNTHESIS The synthesis of glycosaminoglycan by chondrocytes was determined by measuring the incorporation of $\left[{ }^{35} \mathrm{~S}\right]$ sulphate into precipitates 
with cetylpyridinium chloride, according to the method of Kato et al. ${ }^{21}$ Briefly, after the chondrocytes had become confluent, the cells were cultured in fresh medium, with or without muramyl dipeptide, at $37^{\circ} \mathrm{C}$ for 72 hours. The cells were labelled with $37 \mathrm{kBq} / \mathrm{ml}$ of $\left[{ }^{35} \mathrm{~S}\right]$ sulphate for the final six hours of culture. At the end of the labelling period the medium and cells were mixed and digested with pronase E $(2 \mathrm{mg} / \mathrm{ml})$ at $55^{\circ} \mathrm{C}$ for 12 hours. After digestion the precipitates with $1 \%$ cetylpyridinium chloride were collected on a Millipore filter (pore size $45 \mu \mathrm{m}$ ) and the radioactivity retained on the filter was counted in a liquid scintillation spectrometer.

In experiments where two agents (lipopolysaccharide and muramyl dipeptide) were added sequentially, chondrocytes were exposed to the first agent for 24 hours. After removing the medium, the chondrocytes were washed twice with fresh medium and exposed to the second agent for another 24 hours. During the final six hours of culture, the chondrocytes were labelled with $37 \mathrm{kBq} / \mathrm{ml}$ of $\left[{ }^{35} \mathrm{~S}\right]$ sulphate.

\section{Results}

We examined the effect of muramyl dipeptide, lipopolysaccharide, and IL- $1 \alpha$ on the glycosaminoglycan synthesis of cultured rat costal chondrocytes. When rat chondrocytes were incubated in the presence of each agent for 72 hours, lipopolysaccharide and IL-1 $\alpha$ strongly inhibited glycosaminoglycan synthesis in a dose dependent manner; muramyl dipeptide (0.05$50 \mu \mathrm{g} / \mathrm{ml}$ ) had no effect on the glycosaminoglycan synthesis (fig 1). This result was in striking contrast to our finding that in rabbit chondrocytes muramyl dipeptide inhibited glycosaminoglycan synthesis. ${ }^{17}$

Next we examined the possible cooperative effect of muramyl dipeptide and lipopolysaccharide as muramyl dipeptide administered in vivo has been reported to enhance the activities of lipopolysaccharide, such as induction of anaphylactoid shock reaction and lethality ${ }^{22}$ and induction of macrophage colony stimulating

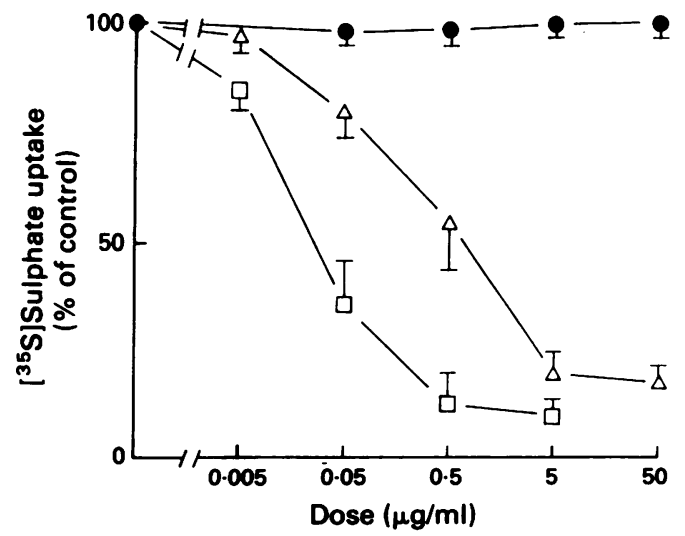

Figure 1 Effects of muramyl dipeptide, lipopolysaccharide, and interleukin l $\alpha$ on the glycosaminoglycan synthesis in cultured rat costal chondrocytes. After reaching confluency rat chondrocytes were cultured for 72 hours with the indicated dose of muramyl dipeptide, lipopolysaccharide, and interleukin Ia respectively. Results are expressed as a percentage of the control value in the absence of agents. muramyl dipeptide; $(\triangle)$ lipopolysaccharide; and $(\square)$ interleukin $1 \alpha$. Values are mean $(S D)$ of four experiments. factor $^{15}$ in mice. Figure 2 shows that muramyl dipeptide $(5 \mu \mathrm{g} / \mathrm{ml})$ strikingly enhanced the lipopolysaccharide induced inhibition of glycosaminoglycan synthesis in rat chondrocytes when muramyl dipeptide and lipopolysaccharide were added simultaneously. This synergistic effect was muramyl dipeptide dose dependent (fig 3). The doses for the half maximal and maximal response were approximately $0.5 \mu \mathrm{g} /$ $\mathrm{ml}$ and $5 \mu \mathrm{g} / \mathrm{ml}$, respectively. There was no evidence of a synergistic effect of muramyl dipeptide in the IL- $1 \alpha$ induced inhibition of glycosaminoglycan synthesis (data not shown).

Five synthetic muramyl dipeptide analogues which are immunoadjuvant active, or inactive ${ }^{10}$ were tested to confirm that the enhancement of the lipopolysaccharide induced inhibition of glycosaminoglycan synthesis is due to muramyl dipeptide itself and not to other contaminations. Figure 4 shows that two immunoadjuvant active muramyl dipeptide analogues, but not inactive muramyl dipeptide analogues, also enhanced lipopolysaccharide induced inhibition of glycosaminoglycán synthesis; hence, the effect of muramyl dipeptide on glycosaminoglycan syn-

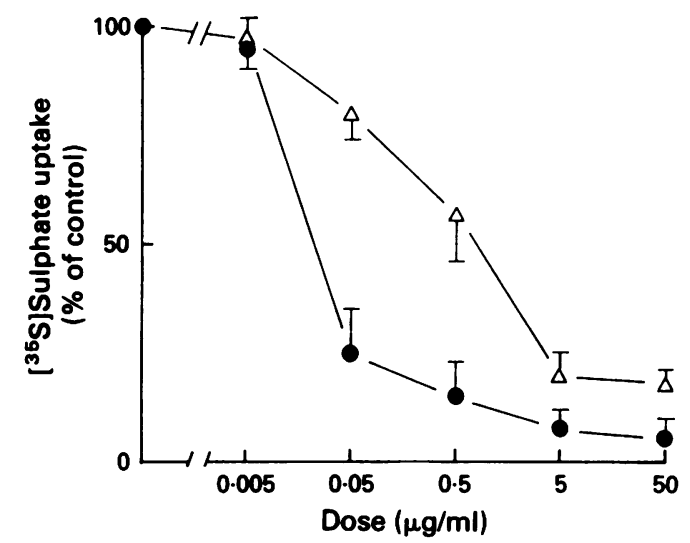

Figure 2 Synergy between muramyl dipeptide and lipopolysaccharide on the inhibition of glycosaminoglycan synthesis in rat chondrocytes. The cultures of confluent chondrocytes were exposed for 72 hours to various doses of lipopolysaccharide alone $(\triangle)$ or lipopolysaccharide plus 5 $\mu g / m l$ muramyl dipeptide $(\mathbf{O})$. Values are mean $(S D)$ of four experiments.

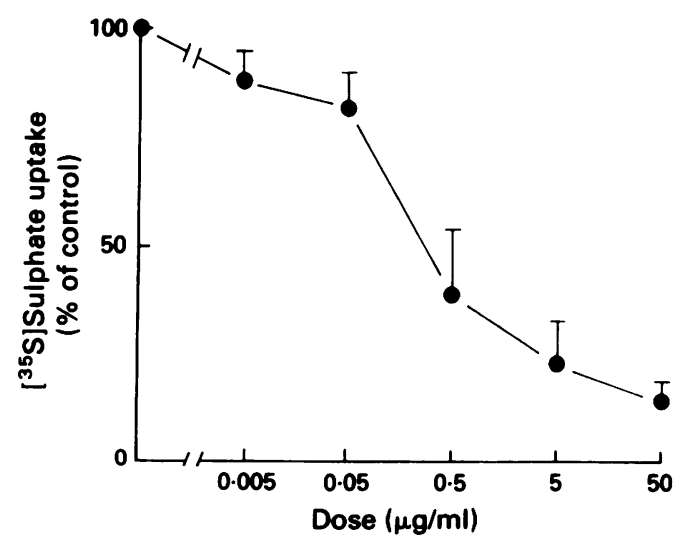

Figure 3 Dose dependency of muramyl dipeptide on the inhibition of glycosaminoglycan synthesis in the presence of lipopolysaccharide. Confluent chondrocytes were cultured for 72 hours in the presence of various doses of muramyl dipeptide plus $0.05 \mu \mathrm{g} / \mathrm{ml}$ lipopolysaccharide. Results are expressed as a percentage of the control value in the presence of lipopolysaccharide $(0.05 \mu \mathrm{g} / \mathrm{ml})$ alone. Values are the mean $(S D)$ of three experiments. 
thesis of rat chondrocytes might parallel the immunoadjuvant activity.

To determine whether the synergism requires the simultaneous presence of lipopolysaccharide and muramyl dipeptide, these agents were added sequentially. When rat chondrocytes were pretreated with muramyl dipeptide $(5 \mu \mathrm{g} /$

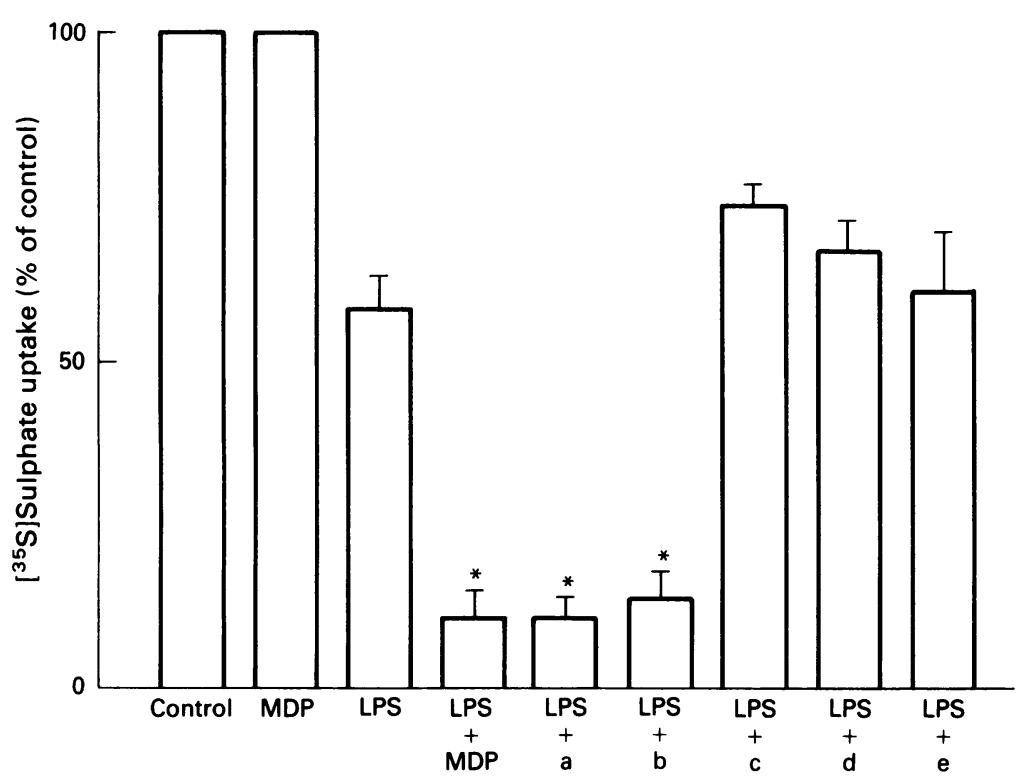

Figure 4 Effect of five synthetic muramyl dipeptide (MDP) analogues on the enhancement of lipopolysaccharide (LPS) induced inhibition of glycosaminoglycan synthesis. Cultured rat chondrocytes were exposed for 72 hours to muramyl dipeptide alone $(10 \mu \mathrm{g} / \mathrm{ml})$,

lipopolysaccharide alone $(0.5 \mu \mathrm{g} / \mathrm{ml})$, lipopolysaccharide plus muramyl dipeptide, or lipopolysaccharide plus each muramyl dipeptide analogue $(10 \mathrm{\mu g} / \mathrm{ml})$. Five analogues of muramyl dipeptide (MurNAc-L-Ala-D-Gln) were (a) MurNAc-L-Ala-D-Glu, (b) MurNAc-L-Ala-D-Gln, (c) MurNAc-L-Ala-L-Gln, (d) MurNAc-L-Ala-L-Glu and (e) MurNAc-L-Ala-D-isoAsn. Results are expressed as a percentage of the control value. Values are the mean $(S D) .{ }^{*} p<0 \cdot 01$ versus lipopolysaccharide alone.

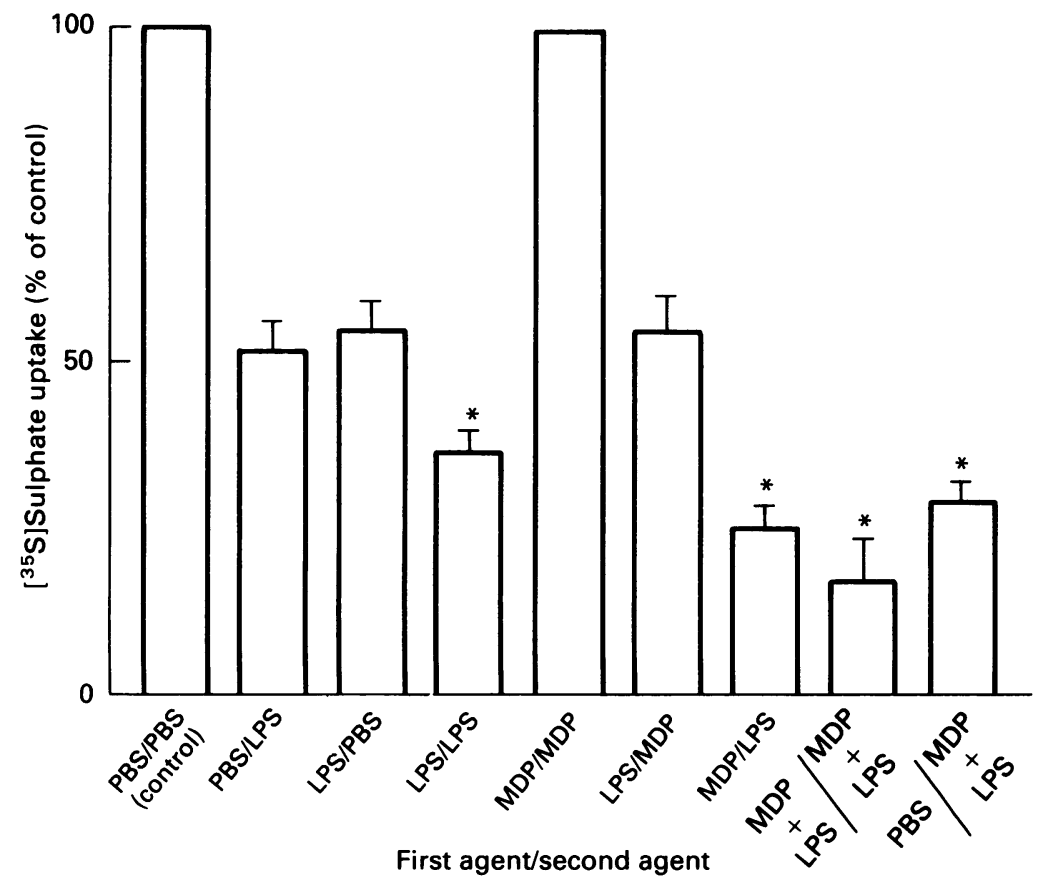

Figure 5 Effect of sequential addition of muramyl dipeptide $(M D P)$ and lipopolysaccharide (LPS) on glycosaminoglycan synthesis. Confluent chondrocytes were exposed to the first agent (lst): muramyl dipeptide alone $(5 \mu \mathrm{g} / \mathrm{ml})$, lipopolysaccharide alone $(0.5 \mu \mathrm{g} / \mathrm{ml})$, or muramyl dipeptide plus lipopolysaccharide. After 24 hours the medium was removed and cells were washed iwice with fresh medium and then cultured in fresh medium containing the second agent $(2 n d)$. The cultures were maintained for the next 24 hours. Phosphate buffered saline $(P B S)$ was used as a control vehicle. Results are expressed as a percentage of the control value $(P B S / P B S)$. Values are the mean $(S D) .{ }^{~} p<0.01$ versus $P B S(I$ st $)$, lipopolysaccharide (2nd).

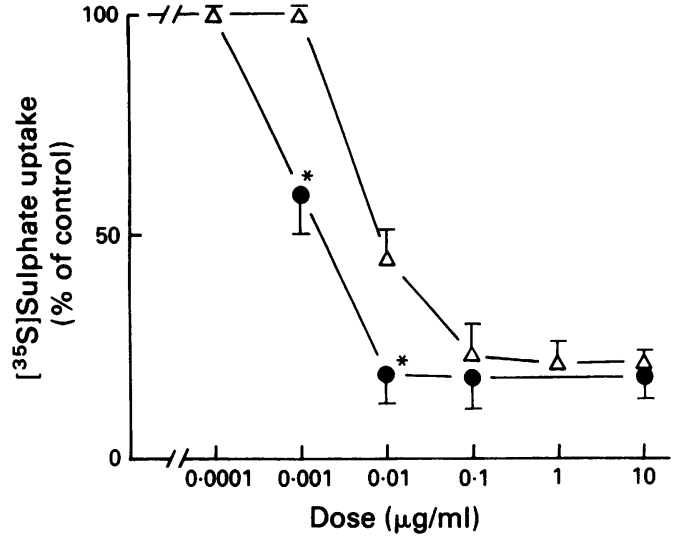

Figure 6 Effect of lipid A on glycosaminoglycan synthesis of rat chondrocytes. After reaching confluency the chondrocytes were exposed for 72 hours to the medium containing various doses of lipid $A$ alone $(\triangle)$, or lipid $A$ plus $5 \mu \mathrm{g} / \mathrm{ml}$ of muramyl dipeptide $(O)$. Values are the mean $(S D)$ of three experiments. ${ }^{*} p<0 \cdot 01$ versus the respective lipid $A$ alone.

$\mathrm{ml}$ ) for 24 hours, washed with fresh medium to remove muramyl dipeptide, and treated with lipopolysaccharide $(0.05 \mu \mathrm{g} / \mathrm{ml})$, glycosaminoglycan synthesis was inhibited to a level comparable with that seen in the simultaneous presence of lipopolysaccharide and muramyl dipeptide throughout the culture (fig 5). In contrast, pretreatment with lipopolysaccharide followed by treatment with muramyl dipeptide was without effect. It seems likely that muramyl dipeptide enhances the susceptibility of rat chondrocytes to lipopolysaccharide.

It seems clear that the lipid A portion of lipopolysaccharide is responsible for a variety of biological activities of lipopolysaccharide. ${ }^{23} 24$ We examined the effects of synthetic $E$ coli lipid A with or without muramyl dipeptide on the glycosaminoglycan synthesis of rat chondrocytes. Figure 6 shows that lipid A as well as lipopolysaccharide inhibited glycosaminoglycan synthesis of rat chondrocytes in a dose dependent manner. The range of doses of lipid A was similar to that of lipopolysaccharide. In addition, when muramyl dipeptide and lipid A were simultaneously added to the medium, the dose response curve of lipid A on the glycosaminoglycan synthesis shifted to the left, hence muramyl dipeptide may enhance the inhibitory effect of the lipid A portion of lipopolysaccharide on glycosaminoglycan synthesis by rat chondrocytes (fig 6).

\section{Discussion}

In this study we found that muramyl dipeptide, an essential structure for the immunoadjuvant activities of bacterial cell wall peptidoglycan, ${ }^{6-10}$ enhanced the lipopolysaccharide induced inhibition of glycosaminoglycan synthesis, whereas muramyl dipeptide alone had no effect on the glycosaminoglycan synthesis. The cooperative effect of the synthetic muramyl dipeptide and lipopolysaccharide was seen with immunoadjuvant active analogues but not with immunoadjuvant inactive analogues. Thus the muramyl dipeptide enhanced inhibition of 
glycosaminoglycan synthesis in the presence of lipopolysaccharide could not be due to contamination.

In cultured rabbit costal chondrocytes, however, muramyl dipeptide without lipopolysaccharide strongly inhibited the glycosaminoglycan synthesis. ${ }^{17}$ There seems to be species differences in the responses to muramyl dipeptide. For example, peritoneal macrophages from guinea pigs and rats (WKA) produced IL-1 and tumour necrosis factor on treatment with muramyl dipeptide in vitro, whereas muramyl dipeptide had no effect on those from mice $(\mathrm{BALB} / \mathrm{c}){ }^{25}$ In the induction of delayed type hypersensitivity such as a corneal reaction, guinea pigs and rabbits were high responders to muramyl dipeptide, but mice $(B A L B / c)$ and SD rats were low responders. ${ }^{26}$ Thus the response of chondrocytes to muramyl dipeptide seems to be dependent on the species. The rabbit chondrocytes may be more sensitive than rat chondrocytes to muramyl dipeptide in the glycosaminoglycan synthesis.

On the other hand, muramyl dipeptide may prime certain animals and cells to respond to the following stimulations even if muramyl dipeptide alone has no direct effect on them. Muramyl dipeptide was reported to enhance the lethal toxicity of lipopolysaccharide in mice when lipopolysaccharide was administered four hours after muramyl dipeptide, ${ }^{22}$ or to prime mice for inducing serum tumour necrosis factor and interferons by the following lipopolysaccharide. ${ }^{27}$ As shown in figure 5, the effect of pretreatment with muramyl dipeptide followed by treatment with lipopolysaccharide was the same as that seen in the case of the simultaneous presence of muramyl dipeptide and lipopolysaccharide, whereas muramyl dipeptide had no effect after pretreatment with lipopolysaccharide. Thus muramyl dipeptide might enhance the susceptibility of rat chondrocytes to lipopolysaccharide. On the other hand, muramyl dipeptide had no synergistic effect on the IL-1 induced inhibition of glycosaminoglycan synthesis (data not shown), thereby suggesting that the effect of muramyl dipeptide might be involved in specific signalling pathways for lipopolysaccharide, but not necessarily other agents. Muramyl dipeptide may possibly increase the number of putative lipopolysaccharide receptors.

The active principle for the endotoxic properties of lipopolysaccharide is confined to a portion designated as lipid A. ${ }^{23}{ }^{24}$ We used a synthetic $E$ coli lipid A instead of $E$ coli derived lipopolysaccharide and it inhibited the glycosaminoglycan synthesis of rat chondrocytes and showed synergism with muramyl dipeptide on the inhibition of glycosaminoglycan synthesis.

Bacterial cell wall components may initiate the inflammatory joint diseases and induce catabolism of articular cartilage. Muramyl dipeptide and lipid A contain immunoadjuvant activity and stimulate macrophages to produce cytokines such as IL-1 and tumour necrosis factor $\alpha$, factors known to degrade the cartilage matrix. ${ }^{34}$ Moreover, Fox et al $^{28}$ reported that muramic acid, a portion of muramyl dipeptide, was detected in joint tissues of rats injected with bacterial cell walls in studies on the pathogenesis of polyarthritis; they thought there may be an interaction between muramyl dipeptide and articular chondrocytes in vivo.

In conclusion, the cooperation between the muramyl dipeptide portion of bacterial cell wall peptidoglycan and the lipid A portion of lipopolysaccharide may play an important part in the initiation of the catabolism of cartilage in inflammatory joint diseases caused by bacterial infection. This culture system of rat chondrocytes may be a useful in vitro model for analysis of the interaction between muramyl dipeptide and lipopolysaccharide.

We thank $\mathrm{K}$ Higuchi for secretarial assistance. Dr Atsuro Inoue (Daiichi Pharmaceutical Co) kindly provided the muramy dipeptide and Drs Tetsuo Shiba and Shoichi Kusumoto (Osak University, Osaka, Japan) provided the muramyl dipeptide analogues. Human recombinant IL-l $\alpha$ was a kind gift of Dainippon Pharmaceuticals.

1 Dinarello C A. Interleukin-1. Rev Infect Dis 1984; 6: 51-95.

2 Beutler B, Cerami A. Tumor necrosis, cachexia, shock, and inflammation: a common mediator. Ann Rev Biochem 1988 7: 505-18.

3 Saklatvala J. Tumor necrosis factor $\alpha$ stimulates resorption and inhibits synthesis of proteoglycan in cartilage. Nature 1986; 32: 547-9.

4 Ikebe T, Hirata M, Koga T. Effects of human recombinan tumor necrosis factor- $\alpha$ and interleukin 1 on the synthesis of glycosaminoglycan and DNA in cultured rat costal hondrocytes. F Immunol 1988; 40: 827-31.

5 Morales T I, Wahl L M, Hascall V C. The effect of bacteria lipopolysaccharides on the biosynthesis and release of proteoglycans from calf articular cartilage cultures. $\mathcal{F}$ Bio Chem 1984; 259: 6702-29.

6 Ellouz F, Adam A, Ciorbaru R, Lederer E. Minima structural requirements for adjuvant activity of bacterial peptidoglycan derivatives. Biochem Biophys Res Commun peptidoglycan deri

7 Kotani S, Watanabe Y, Kinoshita Y, et al. Immunoadjuvan activities of synthetic $\mathrm{N}$-acetyl-myramyl-peptides or -amino acids. Biken Journal 1975; 18: 105-11.

8 Merser C, Sinay P, Adam A. Total synthesis and adjuvan activity of bacterial peptidoglycan derivatives. Biochem Biophys Res Commun 1975; 66: 1317-22.

9 Chedid L, Audibert F, Johnson A G. Biological activities of muramyl dipeptide, a synthetic glycopeptide analogous to bacterial immunoregulating agents. Prog Allergy 1976; 5 : 63-105.

10 Kotani $S$, Tsujimoto $M$, Koga T, Nagao S, Tanaka A, Kawata $S$. Chemical structure and biological activity Kawata S. Chemical structure and biological activity FASEB Y 1986; 45: 2534-40.

11 Nagao S, Tanaka A. Muramyl dipeptide-induced adjuvant arthritis. Infect Immun 1980; 8: 624-6.

12 Koga T, Sakamoto S, Onoue K, Kotani S, Sumiyoshi A. Efficient induction of collagen arthritis by the use of a synthetic muramyl dipeptide. Arthritis Rheum 1980; 23: 993-7.

13 Dewhirst F E. N-Acetylmuramyl dipeptide stimulation of bone resorption in tissue culture. Infect Immun 1982; 35: 133-7.

14 Iribe $H$, Koga $T$, Kotani S, Kusumoto S, Shiba T. Stimulating effect of MDP and its adjuvant-active analogues on guinea pig fibroblasts for the production of thymocyteactivating factor. $\mathcal{F} \operatorname{Exp} M$ Med 1983; 157: 2190-5.

15 Galelli A, Chedid L. Induction of colony-stimulating activity (CSA) by a synthetic muramyl dipeptide (MDP): synergism with LPS and activity in $\mathrm{C} 3 \mathrm{H} / \mathrm{HeJ}$ mice and in endotoxon-tolerized mice. f Immunol 1986; 137: 3211-5.

16 Broudy V C, Kaushansky K, Shoemaker S G, Aggarwal B B, Adamson $\mathbf{J}$ W. Muramyl dipeptide induces production of hemopoietic growth factors in vivo by a mechanism independent of tumor necrosis factor. F I mmunol 1990; 144: independ

17 Ikebe T, Iribe H, Hirata M, Yanaga F, Koga T. Catabolic effects of muramyl dipeptide on rabbit chondrocytes. Arthritis Rheum 1990; 33: 1801-6.

18 Kusumoto S, Tarumi Y, Ikenaka K, Shiba T. Chemical synthesis of $\mathrm{N}$-acetylmuramyl peptides with partial structures of bacterial cell wall and their analogs in relation to immunoadiuvant activities. Bull Chem Soc Jpn 1976; 49: 533-9.

19 Kusumoto S, Okada S, Yamamoto K, Shiba T. Synthesis of 6-0-acyl derivatives of immunoadjuvant active $\mathrm{N}$-acetylmuramyl-L-alanyl-D-isoglutamine. Bull Chem Soc Jpn 1978; 51: 2122-6.

20 Shimomura Y, Yoneda T, Suzuki F. Osteogenesis by chondrocytes from growth cartilage of rat rib. Calcif Tissue chondrocytes from grow

21 Kato Y, Nomura Y, Daikuhara Y, et al. Cartilage-derived factor (CDF) I. Stimulation of proteoglycan synthesis in rat and rabbit costal chondrocytes in culture. Exp Cell Res 1980; 130: 73-8

22 Takada H, Galanos C. Enhancement of endotoxin lethality 
and generation of anaphylactoid reactions by lipopolysaccharides in muramyl-dipeptide-treated mice. Infect Immun 1987; 55: 409-13.

23 Ganalos C, Lüderitz O, Pietschel E T, et al. Synthetic and natural $E$. coli free lipid A express identical endotoxic activities. Eur $\mathcal{X}$ Biochem 1985; 148: 1-5.

24 Homma J Y, Matsuura M, Kanegasaki S, et al. Structura requirements of lipid $A$ responsible for the functions: study with chemically synthesized lipid $A$ and its analogues. f Biochem 1985; 98: 395-406.

25 Nagao S, Akagawa K S, Yamada K, Yagawa K, Tokunaga T, Kotani S. Lack of response of murine macrophages to in vitro activation by muramyl dipeptide (MDP) I. Macrophage activation by MDP is species dependent. Microbiol Immunol 1990; 34: 323-35.

26 Morisaki I. Species and strain differences in responsiveness of bacterial cell walls and their related adjuvant. $\mathcal{F}$ Osaka Univ bacterial cell walls and their related adjuvant. F Osaka Uni
Dent Soc 1980; 25: 229-49. 27 Takada H, Kotani S. MDP and derivatives. In: Stewart-Tull D E S, ed. The theory and practical application

28 Fox A, Schwab J H, Cochran T. Muramic acid detection in mammalian tissues by gas-liquid chromatography-mass spectrometry. Infect Immun 1980; 29: 526-31. 